\title{
Application of Unified Power Flow Controller in Interconnected Power Systems-Modeling, Interface, Control Strategy, and Case Study
}

\author{
Zhengyu Huang, Yixin Ni, C. M. Shen, Felix F. Wu, Shousun Chen, and Baolin Zhang
}

\begin{abstract}
In this paper, a new power frequency model for unified power flow controller (UPFC) is suggested with its dc link capacitor dynamics included. Four principal control strategies for UPFC series element main control and their impacts on system stability are discussed. The main control of UPFC series element can be realized as a combination of the four control functions. The supplementary control of UPFC is added for damping power oscillation. The integrated UPFC model has then been incorporated into the conventional transient and small signal stability programs with a novel UPFC-network interface. Computer tests on a 4-generator interconnected power system show that the suggested UPFC power frequency model and the UPFC- network interface method work very well. The results also show that the suggested UPFC control strategy can realize power flow control fairly well and improve system dynamic performance significantly.
\end{abstract}

\section{INTRODUCTION}

I N RECENT years, new types of FACTS devices have been investigated that may be used to increase power system operation flexibility and controllability, to enhance system stability and to achieve better utilization of existing power systems. The unified power flow controller (UPFC) [1]-[6] is a member of the FACTS family with very attractive features. The development of the first commercial UPFC is being carried out under joint sponsorship of the EPR1, AEP and Westinghouse of USA [7]. Analogue and digital simulators are developed in order to get deeper insight of UPFC mechanism, control strategy and dynarruc performance. In digital simulation, electromagnetic transient programs are widely used for UPFC analysis [8]. However in the study of power system transient and steady-state stability, a power frequency model for UPFC is required to interface UPFC to the quasisteady-state model of ac transmission network and to analyze its effects on large-scale power systems. Some papers [3], [4] suggest UPFC power frequency models which were limited to be used in one machine to infinite bus system. Some other papers [5], [6] developed UPFC power frequency models with its dc link capacitor dynamics neglected or with some other approximations that might lead to undesirable error.

Manuscript received July 27, 1998; revised March 19, 1999. This work was supported in part by RGC (Hong Kong SAR), CRCG of the University of Hong Kong, 'Climb-B' Project, State Sc. and Tech. Commission (China) and EPRI (USA).

Z. Huang, S. Chen, and B. Zhang are with the Dept. of EEE, The Univ. of Hong Kong, Hong Kong SAR.

Y. Ni, C. M. Shen, and F. F. Wu are with the Dept. of EE, Tsinghua University, P. R. China.

Publisher Item Identifier S 0885-8950(00)03824-4.
In this paper, a new power frequency model for UPFC is suggested with its dc link capacitor dynamics included and is suitable to be used in the study of UPFC effects on the real power system dynamic behavior. A novel UPFC-network interface is also developed. In this model the UPFC shunt element control is to keep the UPFC terminal ac bus voltage and the dc link capacitor voltage constant. Four principal control strategies for UPFC series element main control, i. e. line power flow control, series compensation control, transformer-like control, and phase-shifter-like control, and their impacts on system stability are discussed. The final UPFC series element control used can be a combination of the four control outputs through changeable weighting factors. Thus to make full use of UPFC in system control and stability is equivalent to optimize the weights of each control function. The supplementary control of UPFC is added to damp power oscillation along the tie lines after disturbances. The integrated UPFC model has been incorporated into the conventional power system transient and small signal stability programs with the novel UPFC- network interface. Its application is illustrated with a 4-generator interconnected power system [9]. The computer results show that the suggested UPFC power frequency model and the UPFC-network interface method work very well in the study of system dynamic behavior. The results also show that the suggested UPFC control strategy can realize constant power flow control fairly well and can improve system dynamic performance significantly.

\section{MATHEMATICAL MODEL}

\section{A. UPFC Mathematical Model}

Fig. 1 shows a schematic diagram for UPFC, where $n_{1}, X_{t 1}$ and $n_{2}, X_{t 2}$ are the voltage ratio and the reactance of the shunt and series transformers respectively. All the variables used in UPFC model are denoted in Fig. 1 with bold fonts representing phasors.

Per unit system and MKS units are jointly used in modeling. The ac system uses per unit system with its variables calculated based on the system-side $S_{B}$ and $V_{B}$, while the dc variables are expressed in MKS units. We first consider the UPFC dc link capacitor charging dynamics. The dc currents $I_{d 1}, I_{d 2}$ (see Fig. 1) and the capacitor voltage and current have the following relation with harmonics neglected:

$$
\left\{\begin{array}{l}
I_{d}=C \frac{d V_{d}}{d t} \\
I_{d}=I_{d 1}+I_{d 2}
\end{array}\right.
$$




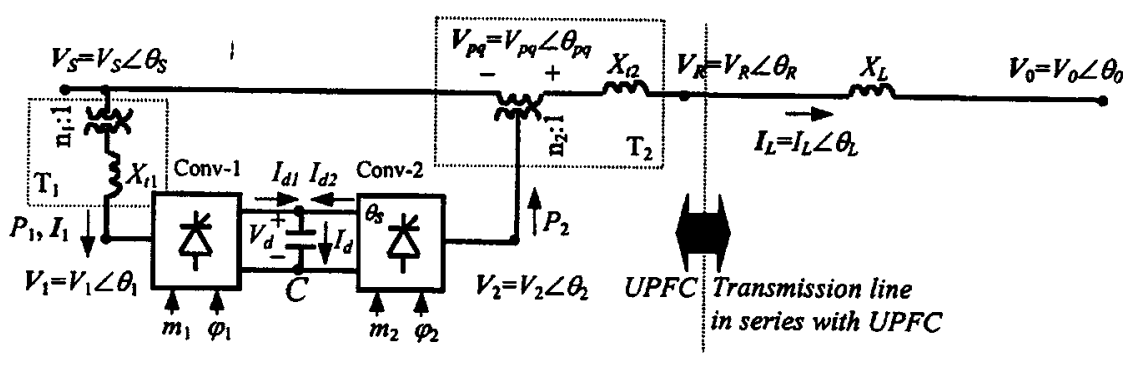

Fig. 1. Transmission line with UPFC installed.

If we assume the inverters are ideal, the real power exchange with the ac system will be ( $P_{1}$ and $P_{2}$ are in p. u.):

$$
\left\{\begin{array}{l}
P_{1}=V_{d} I_{d 1} / S_{B} \\
P_{2}=-V_{d} I_{d 2} / S_{B}
\end{array}\right.
$$

From equations (1) and (2), we have:

$$
C C_{d}=\frac{d V_{d}}{d t}\left(P_{1}-P_{2}\right) S_{B}
$$

From ac system, we know that $P_{1}$ and $P_{2}$ can be calculated by (see Fig. 1):

$$
\left\{\begin{array}{l}
P_{1}=\boldsymbol{R} \boldsymbol{e}\left(\dot{V}_{1} \dot{I}_{1}^{*}\right)=\boldsymbol{R} \boldsymbol{e}\left(\dot{V}_{1}\left(\frac{n_{1} \dot{V}_{S}-\dot{V}_{1}}{j X_{t 1}}\right)^{*}\right) \\
P_{2}=\operatorname{Re}\left(\dot{V}_{p q} \dot{I}_{L}^{*}=\operatorname{Re}\left(\dot{V}_{p q}\left(\frac{\dot{V}_{S}+\dot{V}_{p q}-\dot{V}_{R}}{j X_{t 2}}\right)^{*}\right)\right.
\end{array}\right.
$$

Applying modern PWM control technique[10] to the two voltage source converters, the relations between the inverter dc- and ac-side voltages can be expressed by:

$$
\left\{\begin{array}{l}
V_{1}=m_{1} V_{d} / V_{B} \\
V_{2}=m_{2} V_{d} / V_{B}
\end{array}\right.
$$

where coefficients $m_{1}$ and $m_{2}$ represent the PWM control effects in order to maintain desired inverter ac-side voltages $V_{1}$ and $V_{2}$ respectively. The desired $m_{1}$ and $m_{2}$ are UPFC main control outputs (see Section II-B). $V_{1}$ and $V_{2}$ are in p. u. and $V_{B}$ is the ac system base voltage.

The phase angles of $\dot{V}_{1}$ and $\dot{V}_{2}$ are denoted as $\theta_{1}$ and $\theta_{2}$ respectively. They are controlled through firing angle $\varphi_{1}$ and $\varphi_{2}$ of the two converters and have the relation w. r. t. the phase angle of $\dot{V}_{s}$ as follows:

$$
\left\{\begin{array}{l}
\theta_{1}=\theta_{S}-\varphi_{1} \\
\theta_{2}=\theta_{S}-\varphi_{2}
\end{array}\right.
$$

The desired $\varphi_{1}$ and $\varphi_{2}$ are UPFC main control outputs as well (see Section II-B).

Finally, taking the series transformer ratio into consideration, and rewriting equations (1) (5), the UPFC power frequency model used in dynamic study will be:

$$
C V_{d} \frac{d V_{d}}{d t}=\left(P_{1}-P_{2}\right) S_{B}
$$

$$
\text { where } \begin{aligned}
P_{1} & =\operatorname{Re}\left(\dot{V}_{1}\left(\frac{n_{1} \dot{V}_{S}-\dot{V}_{1}}{j X_{t 1}}\right)^{*}\right), \\
P_{2} & =\operatorname{Re}\left(\dot{V}_{p q}\left(\frac{\dot{V}_{S}+\dot{V}_{p q}-\dot{V}_{R}}{j X_{t 2}}\right)^{*}\right)
\end{aligned}
$$

$$
\begin{aligned}
& V_{1}=m_{1} V_{d} / V_{B} \\
& \theta_{1}=\theta_{S}-\varphi_{1} \\
& V_{p q}=m_{2} V_{d} / V_{B} / n_{2} \\
& \theta_{p q}=\theta_{S}-\varphi_{2}
\end{aligned}
$$

Since there are two network interface (complex) equations, and the desired $m_{1}, \varphi_{1}, m_{2}$ and $\varphi_{2}$ can be obtained from UPFC main control system, therefore based on equation (6) together with UPFC control system equations and ac network interface equations we can analyze UPFC dynamics without difficulty. The first three equations are for state variable $V_{d}$ calculation and the latter 4 equations are for network interface calculation which will be discussed in detail later. It is clear that the UPFC power frequency model derived above can fit various control strategies and multi-machine power system stability analysis.

\section{B. UPFC Main Control}

The UPFC main control includes both shunt and series elements control. A simplified control system block diagram for UPFC is shown in Fig. 2. The shunt element control is shown in Figs. 2(a) and (b): the constant dc link capacitor voltage control realized by controlling the firing angle $\varphi_{1}$ of inverter 1 and the constant UPFC terminal bus voltage control achieved by controlling $m_{1}$ of the PWM controller of inverter 1. PI controller should be used if no control error is allowed. Figs. 2(c) to (f) are the four principal control schemes suggested in [1] for the series element main control. They are constant real and reactive power flow control (Fig. 2(c)), constant series compensation control (Fig. 2(d)), transformer-like control (Fig. 2(e)) and phase-shifter-like control (Fig. 2(f)). The UPFC output series compensation voltage $\dot{V}_{p q}=V_{p q} \angle \theta_{p q}\left(\theta_{p q}=\theta_{2}=\theta_{S}-\varphi_{2}\right)$ can be decomposed as $V_{p}$ and $V_{q}$ (see Fig. 2(h)). The former is perpendicular to $\dot{V}_{S}$ and has strong impacts on real power flow; and the latter is in phase with $\dot{V}_{S}$ and has significant effects on reactive power flow as we all know. From Figs. 2(c) to (f), the desired $V_{p}$ and $V_{q}$ pair corresponding to a specific principal control scheme can be obtained. Then the four pairs of $V_{p}$ and $V_{q}$ will be combined into a final pair of $V_{p}$ and $V_{q}$ through weighting factors (see Fig. 2(g)). After passing through a smooth block the final pair of $V_{p}$ and $V_{q}$ is converted into desired $m_{2}$ and $\varphi_{2}$ for PWM control and firing angle control of inverter 2. It is clear that by setting proper weighting $w_{i}$, the preferred control type(s) will be formed. It can be expected that using optimization theory to generate time varying weighting 


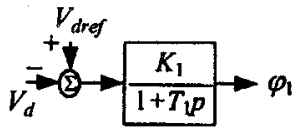

(a)

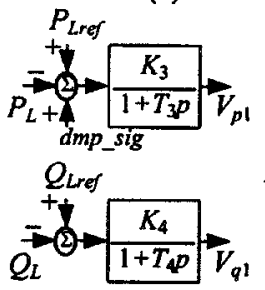

(c)

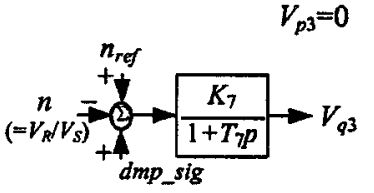

(e)

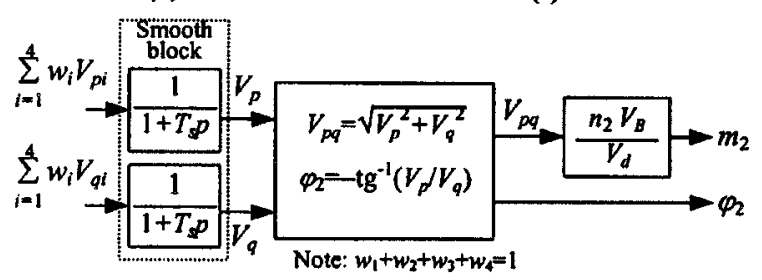

(g)

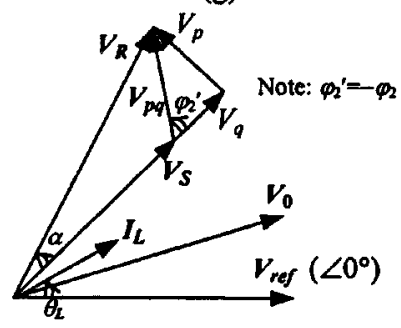

(h)

Fig. 2. The main control and phasor diagram.

factor $w_{i}$, the UPFC could achieve its best steady-state and dynamic performance. At this stage only crispy $w_{i}(1$ or 0$)$ and the simplified first order model is used for the four principal controls. In fact there is no difficulty to include detailed control models.

In Fig. 2(d), $\varphi_{2 \text { ref }}^{\prime}=\pi / 2+\theta_{L}-\theta_{S}$ and $V_{p q r e f}=$ $K_{\text {ref }} I_{L} X_{L}$ are calculated according to the real time measurements of $\left(\theta_{L}-\theta_{S}\right)$ and $I_{L} . K_{r e f}$ is the desired degree of series compensation of the tie line. In phase-shifter-like control, an approximation is made, i.e. assuming the desired $V_{q}$ is zero. The modulation signal $d m p_{-} s g$ in Figs. 2(c) to 2(f) is for damping the power oscillation of the interconnected power system which is the output of UPFC supplementary control and will be discussed in Section III below. In Fig. 2(g), the block of $\frac{n_{2} V_{B}}{V_{d}}$ is derived from equation (6). Fig. 2 and equation (6) constitute the integrated UPFC power frequency model for power system stability analysis. It is clear that there is no limitation to the control system transfer function used. Therefore the concept of user-defined-control [11] can be used for UPFC in order to study the effects of control transfer functions on the integrated power system dynamics.

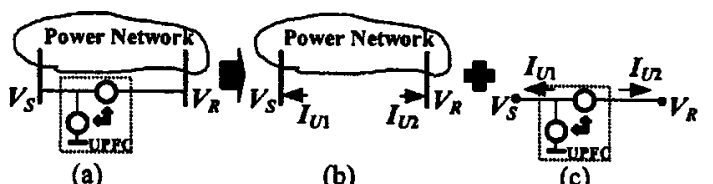

(b)

(c)

Fig. 3. The interface of the UPFC to the network.

\section{Mathematical Models for Other Power System Elements}

The mathematical models for other power system elements are as follows. The subtransient model is used for the generators with simplified third-order excitation control [12]. The mechanical power of each generator is taken as a constant. Loads are expressed as constant impedance and the ac network is linear. In order to simplify the software development, we assume $X_{d}^{\prime \prime}=$ $X_{q}^{\prime \prime}=X^{\prime \prime}$ for generators so that $X^{\prime \prime}$ can be included in the bus admittance matrix, and the latter can be reduced to generator internal buses [12] and used for ac network interface to UPFC which will be discussed in detail below.

\section{Interface of UPFC to the AC Network}

The interface calculation of UPFC to ac network will have significant impacts on transient stability analysis speed and accuracy. The sequential solution method, which has been successfully used for ac-dc interface in ac/dc power system load flow and transient stability analysis, has been extended here for UPFC interface to ac network (see Fig. 3).

In the interface calculation we assume that the bus admittance matrix has been reduced to generator internal buses with UPFC ac terminal buses remained. The corresponding reduced bus admittance matrix equation takes the form:

$$
\left[\begin{array}{cc}
Y_{G G} & Y_{G U} \\
Y_{U G} & Y_{U U}
\end{array}\right]\left[\begin{array}{c}
\dot{E}_{G} \\
\dot{V}_{U}
\end{array}\right]=\left[\begin{array}{l}
\dot{I}_{G} \\
\dot{I}_{U}
\end{array}\right]
$$

where $\dot{E}_{G}$ : generator intemal voltage behind the subtransient reactance $X^{\prime \prime} . \dot{V}_{U}=\left[\dot{V}_{S}, \dot{V}_{R}\right]$ : ac terminal bus voltages of the UPFC (see Fig. 3). $\dot{I}_{G}$ : generator currents injecting to the network. $\dot{I}_{U}=\left[\dot{I}_{U 1}, \dot{I}_{U 2}\right]$ : UPFC currents injecting to the network (see Fig. 3).

The UPFC currents injecting to the ac network can be expressed by (see Figs. 1 and 3):

$$
\left\{\begin{array}{l}
\dot{I}_{U 1}=-\frac{n_{1} \dot{V}_{S}-\dot{V}_{1}}{j X_{t 1}} n_{1}-\frac{\dot{V}_{S}+\dot{V}_{p q}-\dot{V}_{R}}{j X_{t 2}} \\
\dot{I}_{U 2}=\frac{\dot{V}_{S}+\dot{V}_{p q}-\dot{V}_{R}}{j X_{t 2}}
\end{array}\right.
$$

Suppose all the state variables at $t=t_{n+1}$ have been predicted according to the numerical integration and now we are going to make network solution for algebraic variables. It is clear that in equation (8), though the UPFC output voltage magnitudes $V_{1}$ and $V_{p q}$ can be known from control output and equation (6), the phase angles of $\dot{V}_{1}$ and $\dot{V}_{p q}$ are unknown since they depend on the phase angle of $\dot{V}_{S}$. The phase angle of $\dot{V}_{S}$ is unknown that should be obtained from the network solution. Therefore an iteration approach is required to obtain the network solution. 
The iteration is conducted as follows. Substituting equation (8) into equation (7), and rearranging the second equation of equation (7), we finally have:

$$
\left\{\begin{array}{l}
\dot{I}_{1 G}+\dot{I}_{2 G}+\left(Y_{s s}+Y_{R S}+\frac{n_{1}^{2}}{j X_{t 1}}\right) \dot{V}_{S}+\left(Y_{S R}+Y_{R R}\right) \dot{V}_{R} \\
\quad=\frac{n_{1}}{j X_{t 1}} \dot{V}_{1} \\
\dot{I}_{2 G}+\left(Y_{R S}-\frac{1}{j X_{t 2}}\right) \dot{V}_{S}+\left(Y_{R R}+\frac{1}{j X_{t 2}}\right) \dot{V}_{R}=\frac{1}{j X_{t 2}} \dot{V}_{p q}
\end{array}\right.
$$

where $\left[\begin{array}{cc}Y_{S S} & Y_{S R} \\ Y_{R S} & Y_{R R}\end{array}\right]=Y_{U U}$, and $\left[\begin{array}{l}\dot{I}_{1 G} \\ \dot{I}_{2 G}\end{array}\right]=Y_{U G} \dot{E}_{G}$ which can be calculated according to generator state variables. If we define a constant matrix:

$$
\tilde{Y}_{U U}=\left[\begin{array}{cc}
\left(Y_{S S}+Y_{R S}+\frac{n_{1}^{2}}{j X_{t 1}}\right) & \left(Y_{S R}+Y_{R R}\right) \\
\left(Y_{R S}-\frac{1}{j X_{t 2}}\right) & \left(Y_{R R}+\frac{1}{j X_{t 2}}\right)
\end{array}\right]
$$

and a fictitious time varying current vector:

$$
\tilde{I}_{U}=\left[\begin{array}{c}
\frac{n_{1}}{j X_{t 1}} \dot{V}_{1}-\left(\dot{I}_{1 G}+\dot{I}_{2 G}\right) \\
\frac{1}{j X_{t 2}} \dot{V}_{p q}-\dot{I}_{2 G}
\end{array}\right]
$$

we have:

$$
\tilde{Y}_{U U} \dot{V}_{U}=\tilde{I}_{U}
$$

The equations (10) to (12) are used for the iteration of UPFCnetwork interface as follows:

Step 1: Estimate the initial voltages of $\dot{V}_{S}^{(0)}$ and $\dot{V}_{R}^{(0)}$ (e. g. according to the last step) and calculate $\widetilde{I}_{U}$ based on equations (11) and (6).

Step 2: Solve equation (12) for $\dot{V}_{U}=\left(\dot{V}_{S}^{(1)}, \dot{V}_{R}^{(1)}\right)^{\prime}$. If the difference of $\left(\dot{V}_{S}^{(1)}, \dot{V}_{R}^{(0)}\right.$ and $\left(\dot{V}_{S}^{(0)}, \dot{V}_{R}^{(0)}\right)$ is less than the given tolerance then $\dot{V}_{S}^{(1)}$ and $\dot{V}_{R}^{(1)}$ are considered as the solution of equation (9). Otherwise go to step 3

Step 3: Update $\left(\dot{V}_{S}^{(0)}, \dot{V}_{R}^{(0)}\right)$ by $\left(\dot{V}_{S}^{(1)}, \dot{V}_{R}^{(1)}\right)$ and repeat steps 1 and 2 till convergence is reached.

It is clear that Step 1 corresponds to the UPFC calculation with estimated terminal voltages (see Fig. 3(c)). Step 2 corresponds to ac network solution with UPFC effect represented as an injecting current to ac network (see Fig. 3(b)) except that we defined a fictitious current vector as the UPFC-network interface variable to save CPU time. After the solution of interface voltages $\dot{V}_{U}=\left(\dot{V}_{S}, \dot{V}_{R}\right)^{\prime}$ the first part of matrix equation (7) is used for calculation of generator injecting current $\dot{I}_{G}$.

By this iteration approach there is no need to rewrite the entire program. The stability analysis can be realized simply by inserting just a few new subroutines into the existing software. The network solution accuracy can be controlled via the interface tolerance setting. This interface can also be extended and used for other FACTS devices.

\section{Discussion OF UPFC SERIES ElEMENT MAIN CONTROL SCHEME}

As suggested in [1] there are four principle control schemes for the series compensation voltage $\dot{V}_{p q}$ i. e. constant real and reactive power flow control constant series compensation control phase-shifter-like control and transformer-like control. At the normal operation the constant real and reactive power control is the most attractive especially for the tie line power flow control. However under a large disturbance such as a three phase fault this control trying to keep constant power flow over the tie line might not be appropriate for system transient stability. What is the best control strategy for the first swing stability is our current concern.

In a one-machine-to-infinite-bus system according to equal area criterion it is well known that the best control in the first swing is the one which can lead to the largest electric power transfer from generator to receiving end and hence the largest rotor deceleration power.

For the constant real and reactive power control an effficient way to improve first swing stability is fast increasing power reference for a certain period which can lead to fast power transfer so as to release rotor kinetic energy. However it is difficult to know the optimal reference power and optimal time duration because the fault severity is unforeseeable. The phase-shifter-like control will not change the shape of the power-angle curve if we consider the phase-shifter-like control is equivalent to a constant generator power angle shift during the first swing, and the total available deceleration area has no significant change. So this control is not so effcient in improving system transient stability. The transformer-like control is basically a voltage control which may increase the peak of the power-angle curve by $10-20 \%$ in order to avoid overvoltage. The total deceleration area increase is still not so good. For the constant series compensation control, if compensation degree $K_{\text {ref }}$, is high enough (here we assume the inverter has enough capacity), the power-angle characteristics can rise significantly via reducing tie line apparent reactance which is clearly beneficial to the system transient stability.

Based on the discussion above we can see that during the first swing, there are mainly two approaches to improve first swing stability. One is fast increasing of power reference for a certain period, and the other is to switch the series element control from constant power control to constant series compensation control. However when the rotor reaches the maximum angle in the first swing, the constant series compensation control should be switched to some other control, otherwise this control will over-decelerate the rotor and lead to undesirable large power oscillation in the subsequent swings.

In the subsequent swings, supplementary control will play an important role in damping the power oscillation on the tie lines. The supplementary control can be applied to the shunt element control via voltage modulation or to the series element via power modulation, voltage modulation, phase-shift-angle modulation or series compensation degree modulation depending on the control strategy currently used. In order to avoid unnecessary switching of control strategy, right after the first swing the control returns to the constant real and reactive power control. Therefore two different damping schemes are tested in this paper. One is the voltage modulation in voltage control of the shunt element (see $d m p_{-} s i g$ in Fig. 2(b)). The other is power modulation in constant power control of the series element (see $d m p_{-} s i g$ in Fig. 2(c)). In our paper the input signal used for supplementary control is the tie line real power flow or its terminal voltage phase angle difference. The supplementary control has a PSS-like transfer function (see Fig. 4) with its output signal connected to the main control shown in Fig. 2(b) or (c). 


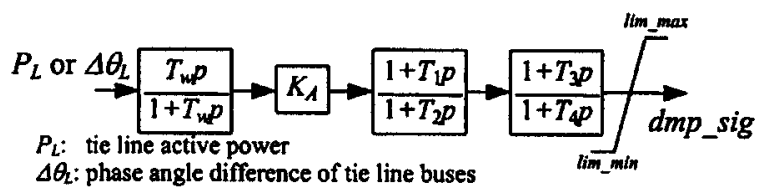

Fig. 4. Supplementary control block diagram.

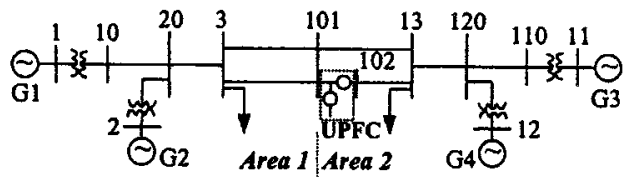

Fig. 5. The computer test system diagram.

As a summary the control scheme used in our paper is as follows: the constant real and reactive power control is used for the series element control at the steady state. It will be switched to constant series compensation control with higher compensation degree during the first swing. The switching can be triggered by large disturbance signal (such as $\Delta R_{L} / \Delta t$ ). The series element control will return to the constant power control right after the peak point of the first swing. The shunt element control is constant UPFC ac bus voltage control together with constant dc link capacitor voltage control. The supplementary control is added on the shunt element via voltage modulation or added on the series element via real power modulation. The control system output variables are $m_{1}, m_{2}, \varphi_{1}$ and $\varphi_{2}$.

\section{Computer Test Results}

\section{A. Test System and Studied Cases}

A 4-generator 2-area interconnected power system [9] is used for the computer test (see Fig. 5). At the steady state, the electric power output of generators 1 to 4 is $700 \mathrm{MW}, 700 \mathrm{MW}$, $716 \mathrm{MW}$, and $700 \mathrm{MW}$ respectively. The loads on the buses 3 and 13 are $967 \mathrm{MW}$ and $1767 \mathrm{MW}$. About $390 \mathrm{MW}$ power is transferred from area 1 to area 2 with a UPFC installed on one of the tie lines. The generator, exciter and load models are the same as described in Section II-C. The disturbance used is a three-phase stub fault on bus 3 at $t=0.50$ second, and cleared in 0.10 second. Three groups of cases are tested, i.e., group 1: system first-swing transients without supplementary control of UPFC; group 2: supplementary control (S. C.) effects on system dynamic behavior; and group 3: suggested control strategy and its effect on system dynamic behavior.

All the studied cases are listed in Table I with corresponding case ID and conditions. The control parameters are presented in the appendix. The computer test results of each group are described below.

\section{B. Computer Results of the First Swing Transients}

The computer test results for case 1a (without UPFC) are shown in Fig. 6, where Fig. 6(a) is the rotor angle plot and Fig. 6(b) is real power on line 102-13. Figs. 7 and 8 are for cases $1 \mathrm{~b}$ (UPFC with constant tie line $P$ and $Q$ control) and 1c (UPFC with constant series compensation control) respectively.
TABLE I

TEST CASE ID AND THE CORRESPONDING CONDITIONS

\begin{tabular}{|c|c|c|c|c|c|}
\hline Group \# & Case ID & UPFC & Main Ctrl. & Supp. & Ctrl. \\
\hline 1 & la & $\mathrm{N}$ & -.. & Sig. IV & d. $\mathrm{El}$ \\
\hline & $1 \mathrm{~b}$ & $\mathrm{Y}$ & Const. $\mathrm{P} / \mathrm{Q}$ & $\ldots$ & ..- \\
\hline & 1c & $\mathrm{Y}$ & Const. C. $\left(\mathrm{K}_{\mathrm{ret}}=0.4\right.$ & 0.4) --- & --- \\
\hline 2 & $2 a$ & $\mathrm{Y}$ & Const. P/Q & $\mathrm{P}_{\mathrm{L}}$ & shunt \\
\hline & $2 \mathrm{~b}$ & Y & Const. P/Q & line $\theta$ diff. & shunt \\
\hline & $2 c$ & $\mathrm{Y}$ & Const. P/Q & $\mathrm{P}_{\mathrm{L}}$ & series \\
\hline & $2 \mathrm{~d}$ & 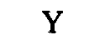 & Const. P/Q & $e \theta$ diff. & series \\
\hline 3 & 3 & $\mathrm{Y}$ & * & $P_{L}$ & shunt \\
\hline
\end{tabular}

- see suggested control strategy at the end of Section 3

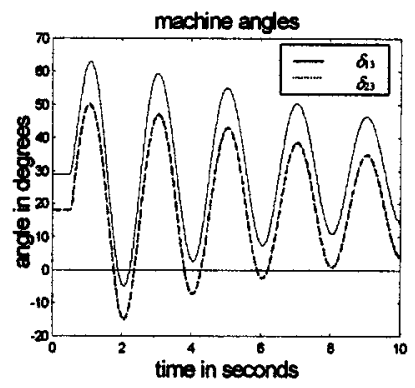

(a)

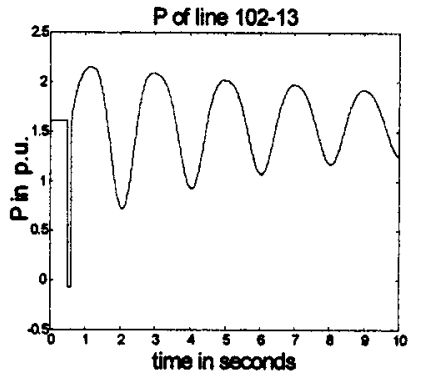

(b)
Fig. 6. Plots of case 1a.

The figures include (a) rotor angles, (b) voltages of the fault bus 3 and UPFC sending-end bus 101, (c) UPFC-installed tie line real power and (d) dc capacitor voltage $V_{d}$. The following observations can be made:

- When UPFC has constant power flow control, the first swing peak angle (Fig. 7(a)) is almost the same as and even a little bit worse than case 1a (Fig. 6(a)) in which there is no UPFC in the system. This means the constant power flow control might have negative effect on system first swing transient if there is no fast reference power increase during the period.

- When UPFC has constant series compensation control, the first swing peak angle reduced noticeably (Fig. 8(a)) which is consistent to our discussion. We can also see that the line power increases greatly in the first swing (Fig. 8(c)) to fast release the rotor kinetic energy.

- After first swing, the constant power flow control can recover the controlled tie line power to the setting point very quickly (Fig. 7(c)) which is extremely desirable. However the constant series compensation control case will have long period of power oscillation on the controlled tie line (Fig. 8(c)).

- The time simulation shows very good convergence in UPFC-network interface which proves that the suggested UPFC model and interface method work very well in transient stability analysis.

- The dc capacitor voltage fluctuation during transient (Figs. $7(\mathrm{~d})$ and $8(\mathrm{~d})$ ) is less than $0.3 \mathrm{kV}$, about $1.5 \%$ of its 

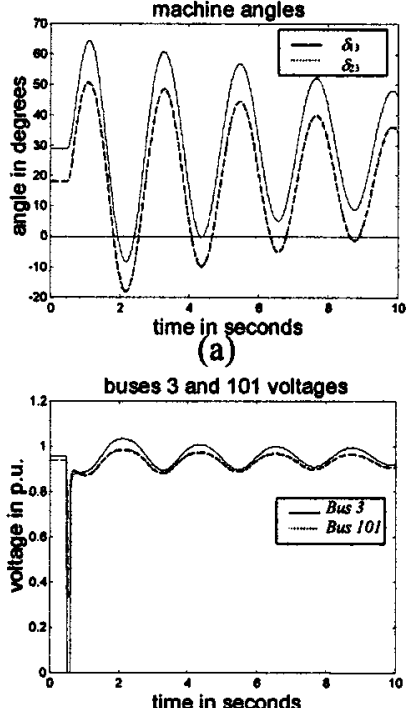

(b)

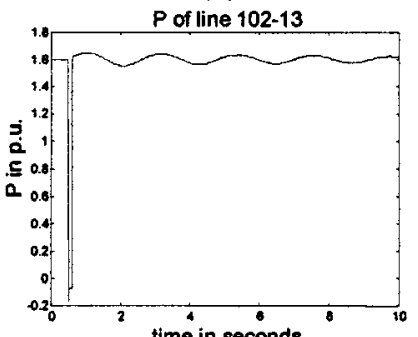

(c)

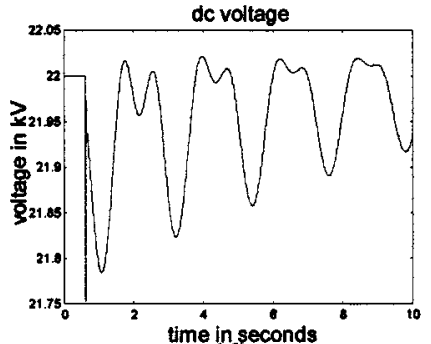

(d)

Fig. 7. Plots of case $1 b$.

rated value $22 \mathrm{kV}$, which means to neglect dc link capacitor dynamics may cause some error but it is acceptable to certain extent.

- The rotor angle plots in Figs. 7 and 8 also show that system damping is very poor and supplementary damping control is necessary.

\section{Effects of UPFC Supplementary Control}

Four types of supplementary control are tested. They are cases $2 \mathrm{a}$ to $2 \mathrm{~d}$ (see Table I) and all with constant power flow control for the series element. The differences are the input signal of supplementary control and the modulation mode (see Table I). Only curves for cases $2 \mathrm{a}$ and $2 \mathrm{c}$ are presented in Fig. 9. The computer results show:

- The parameters of supplementary control for cases $2 \mathrm{a}$ to $2 \mathrm{~d}$ are properly designed to get desired damping effect for the interarea mode. Therefore for all the four cases, the system damping is greatly improved (see Figs. 9(a) for

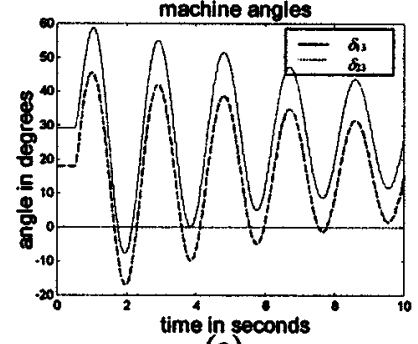

(a)

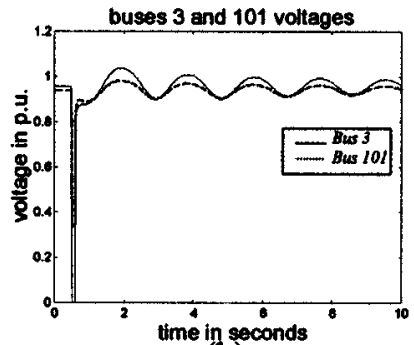

(b)

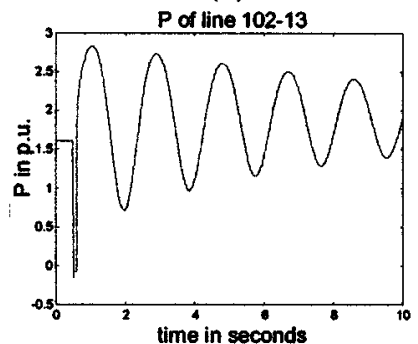

(c)

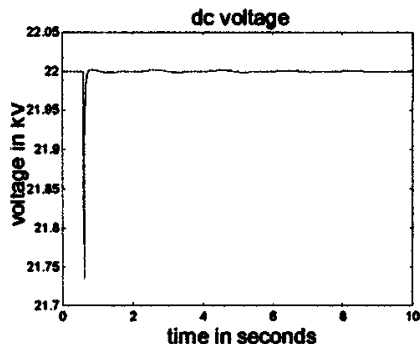

(d)

Fig. 8. Plots of case 1c.

case 2a and Fig. 9(c) for case 2c as two examples) which means the input signal, transfer function, parameters of supplementary control are well selected and the modulation mode is effective.

- For all the four cases the input signal for supplementary control $\left(\Delta P_{L}\right.$ or $\left.\theta_{L}\right)$ has similar 'shape' in time domain since $\Delta P_{L} \approx \frac{V_{L L} V_{L 2}}{X_{L}} \sin \theta_{L} \approx K \theta_{L}$ (if $\theta_{L}$ is not very large), where $V_{L 1}$ and $V_{L 2}$ are sending and receiving end voltages of the tie line. Besides the transfer functions used are similar. Therefore the output of supplementary control signal is quite similar to each other (see Figs. 9(b) for case 2a and Fig. 9(d) for case $2 \mathrm{c}$ as two examples). The supplementary control output limits used are $0.2 \mathrm{p}$. u. for voltage modulation for avoiding over-voltage and $0.5 \mathrm{p}$. u. for power modulation for avoiding overload.

- The interarea mode for each case is as follows:

$$
\text { w/o S. C.: } \quad-0.0808+j 2.8896
$$




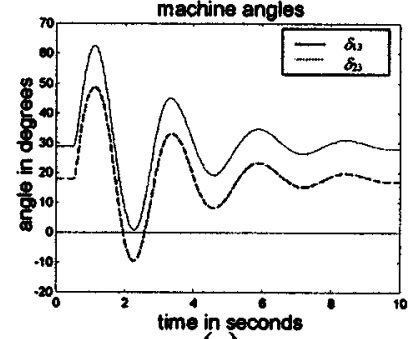

(a)

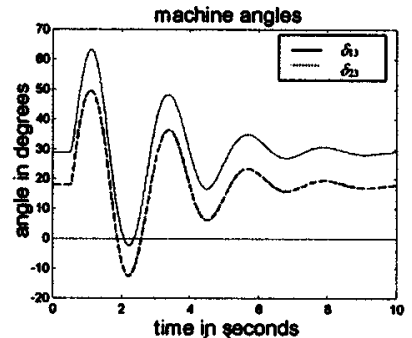

(c)

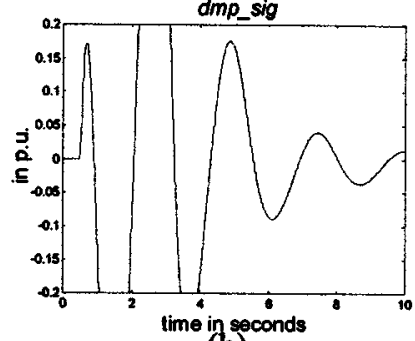

(b)

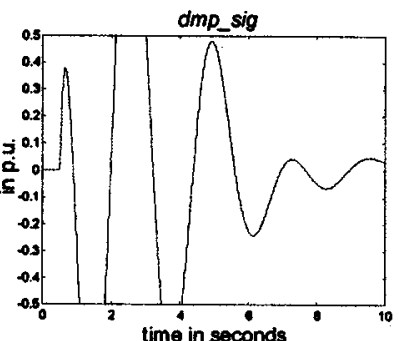

(d)
Fig. 9. Effects of supplementary control.

$$
\begin{aligned}
& \text { Case 2a (shunt- } \left.P_{L}\right):-0.4306+j 2.4779 \\
& \text { Case 2b (shunt- } \theta): \quad-0.5340+j 2.6586 \\
& \text { Case 2c (series- } \left.P_{L}\right):-0.6174+j 2.7401 \\
& \text { Case 2d (series- } \theta \text { ): } \quad-0.6214+j 2.8915
\end{aligned}
$$

From eigenvalue analysis we can see the interarea damping has been improved significantly after supplementary control is added.

\section{Computer Results of Suggested Control Strategy}

From the above test and study, it can be seen that the performance of the UPFC is greatly affected by its control strategy. Computer results till now are consistent to our discussion in Section III. Now we test the suggested UPFC control scheme, i. e. constant power flow control at the steady state and after the first swing, constant series compensation control during first swing. The supplementary control is voltage modulation on shunt element control with tie line real power as input signal. The corresponding computer results are shown in Fig. 10. The plots include: (a) rotor angle, (b) buses 3 and 101's voltages, (c) real power flow of the two tie lines, (d) dc link capacitor voltage, (e) exchange power of inverters 1 and 2 with ac system, and (f) voltages $V_{1}$ and $V_{p q}$. From the plots, we can see that the first swing, the system damping and the power flow control after large disturbance are all quite satisfactory. We can also see that the $V_{d}$ fluctuation is very small and the power exchange of inverters with ac system is at reasonable level. The $V_{1}$ and $V_{p q}$ are also in the acceptable range. All the facts show that the suggested control scheme works very well in the system transients and can control interarea power flow effciently and improve system dynamic behavior significantly.

\section{CONCLUSION}

The suggested UPFC power frequency model and the developed UPFC-network interface method work very well

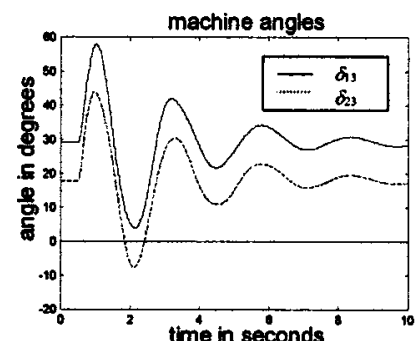

(a)

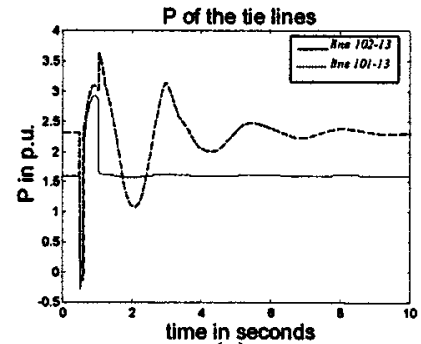

(c)

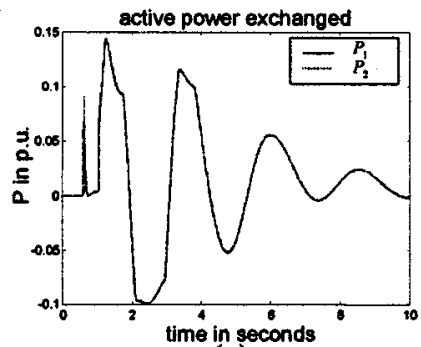

(e)

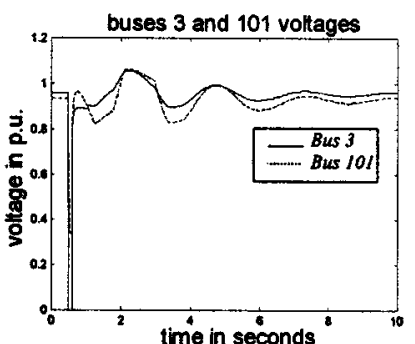

(b)

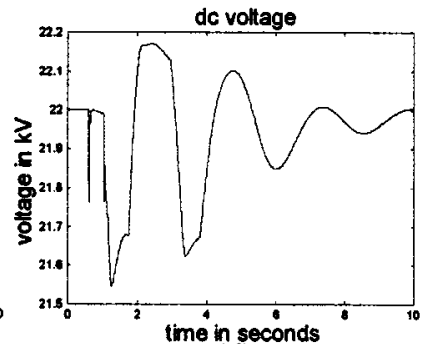

(d)

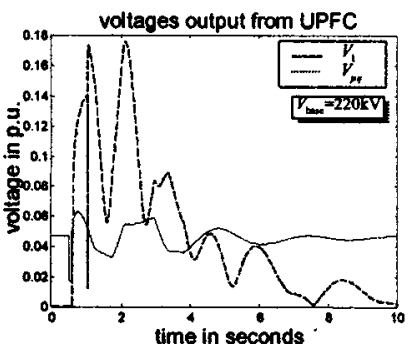

(f)
Fig. 10. Results of the suggested control scheme.

in the study of power system dynamics with satisfied convergence and accuracy. Four principal main control strategies are discussed and the computer tests results support the discussion conclusion very well. The constant power flow control is good for steady state control and the constant series compensation control is useful for first swing stability. The supplementary control is very efficient in damping intcrarea power oscillation. The suggested UPFC control can realize the desired control strategy flexibly and improve system dynamic performance significantly.

\section{APPENDIX}

The parameters of UPFC:

$$
\begin{aligned}
& V_{B}=220 \mathrm{kV}, S_{B}=100 \mathrm{MVA}, n_{1}=0.05, n_{2}=0.25, \\
& X_{t 1}=0.00025, X_{t 2}=0.05 \text { (all in p. u. of } 220 \mathrm{kV} \text { base). }
\end{aligned}
$$

The parameters of UPFC main control:

$$
\begin{aligned}
& K_{1}=0.05, T_{1}=0.01, K_{2}=1.0, T_{2}=0.05, K_{3}=5.0, \\
& T_{3}=0.1, \\
& K_{4}=5.0, T_{4}=0.1, T_{5}=0.02, T_{6}=0.02, K_{\text {ref }}=0.4, \\
& V_{d r e f} \approx 22 \mathrm{kV} .
\end{aligned}
$$


TABLE II

THE SuPPlementary CONTROL PARAMETERS (T IN Sec.)

$\begin{array}{ccccccc}\text { Case ID } & K_{A} & T_{w} & T_{1} / T_{3} & T_{2} / T_{4} & \text { lim_max } & \text { lim_min } \\ \text { 2a } & 0.83 & 3.0 & 0.02 & 0.33 & 0.2 & -0.2 \\ \text { 2b } & 2.83 & 3.0 & 0.02 & 0.23 & 0.2 & -0.2 \\ \text { 2c } & 0.83 & 3.0 & 0.02 & 0.19 & 0.5 & -0.5 \\ \text { 2d } & 7.17 & 3.0 & 0.02 & 0.23 & 0.5 & -0.5\end{array}$

\section{REFERENCES}

[1] L. Gyugyi, "Unified Power-Flow Control Concept for Flexible AC Transmission Systems," IEE Proceedings-C, vol. 139, no. 4, pp. 323-331, July 1992.

[2] I. Papic, P. Zunko, and D. Povh, "Basic Control of Unified Power Flow Controller," IEEE Trans. on Power Systems, vol. 12, no. 4, pp. 1734-1739, Nov. 1997.

[3] R. Mihalic, P. Zunko, and D. Povh, "Improvement of Transient Stability Using Unified Power Flow Controller," IEEE Trans. on Power Delivery, vol. 11, no. 1, pp. 485-491, Jan. 1996.

[4] K. S. Smith, L. Ran, and J. Penman, "Dynamic Modeling of a Unifed Power Flow Controller," IEE Proc.-Gener. Transm. Distrib., vol. 144, no. 1, pp. 7-12, Jan. 1997.

[5] M. Noroozian, L. Angquist, and M. Ghandhari, et al., "Improving Power System Dynamics by Series-connected FACTS devices," IEEE Trans. on Power Delivery, vol. 12, no. 4, pp. 1635-1641, Oct. 1997.

[6] S. Limyingcharoen, U. D. Annakkage, and N. C. Pahalawaththa, "Effects of unified powerflow controllers on transient stability," IEE Proc.Gener. Transm. Distrib., vol. 145, no. 2, pp. 182-188, March 1998.

[7] A. S. Mehraban and A. J. F. Keri, et al., "Application of the World's First UPFC on the AEP System," in EPRI-the Future of Power delivery Conference, Washington, DC, 1996.

[8] M. P. Bottino, B. Delfino, and G. B. Denegri, "FACTS for More Effective Networks: Unified Power Flow Controller Using PSCAD/EMTDC Code," in EPRI-the Future of Power delivery Conference, Washington, DC, 1996.

[9] P. Kundur, Power System Stability and Control: McGraw-Hill Inc., 1994, pp. 813-816.

[10] N. Mohan, T. M. Undeland, and W. P. Robbins, Power Electronics: Converters, Applications and Design, Second ed: John Wiley \& Sons, 1995.

[11] EPRI Report, "Extended transient-midterm stability program (ETMSP),", EPRI TR-102 004-V2R1, 1994.

[12] P. Anderson and A. A. Fouad, Power system control and stability: IEEE Press, 1994.
Zhengyu Huang $\mathrm{Ph}$. D. candidate in Tsinghua University and currently research assistant of the University of Hong Kong. His major research area is FACTS, Fuzzy logic theory applications in power system, power system control and stability.

Yixin Ni received her B. Eng., M. Eng., and Dr. Eng. from Electrical Engineering Department, Tsinghua University, P. R. China in 1968, 1981 and 1983 respectively. Her research interest is in power system modeling, shnulation, stability and control, and power electronics applications in power systems. She was a professor of Tsinghua University and is now with The University of Hong Kong.

C. M. Shen received his B.Sc. (Eng.) and M.Sc. (Eng.) degrees in EE from the University of Hong Kong (HKU) in 1963 and 1965 respectively and his Ph.D. degree in EE from Queen Mary College, University of London in 1969. Since then he has joined the HKU. His research interest is in Load flow, fault analysis, state estimation and stability and control of power systems. He is an executive committee member of the Specialized Section in Power, IKE Hong Kong Centre.

Felix F. Wu joined the University of Hong Kong as Chair professor of Electrical Engineering in September 1995 and is now Pro-vice-chancellor of HKU. Prior to that, he was Professor and Vice Chairman, Department of Electrical Engineering and Computer Sciences, University of California, Berkeley, the same institute where he received his Ph.D. degree. His research interests are in power system planning and operation, including economics and reliability in system planning, real-time security assessment, and design of energy management systems and distribution automation. Recently he has been involved in the design of industry restructuring and electricity pricing.

S. S. Chen received his B. Eng. degree in EE Dept., Tsinghua University (Beijing, China) in 1955. Since then, he has been working in Tsinguha University. He is currently a professor in EE Dept. His research interest is in power system modeling, analysis, HVDC transmission, FACTS and distribution automation.

B. L. Zhang reed his B. Eng. degree in EE Dept., Tsinghua University (Beijing, China) in 1950. Since then, he has been working in Tsinguha University. He is currently a retired professor in EE Dept. His research interest is in power system modeling, simulation, protection, HVDC transmission, FACTS and power system stability and control. 